\title{
Inhibition of Mitochondrial Fission Preserves Photoreceptors after Retinal Detachment
}

\author{
Xiangjun She, ${ }^{*}$ Xinmin Lu, ${ }^{*}$ Tong $\mathrm{Li}_{,}{ }^{*}$ Junran Sun, ${ }^{*}$ Jian Liang, ${ }^{* \dagger}$ Yuanqi Zhai, ${ }^{\dagger \dagger}$ Shiqi Yang, ${ }^{*}$ Qing Gu,${ }^{\ddagger}$ Fang Wei, \\ Hong Zhu, ${ }^{* \dagger}$ Fenghua Wang, ${ }^{* \dagger}$ Xueting Luo, ${ }^{* \star}$ and Xiaodong Sun ${ }^{* \dagger \ddagger}$
}

From the Department of Ophthalmology, * Shanghai General Hospital (Shanghai First People's Hospital), Shanghai Jiao Tong University School of Medicine, Shanghai; the Shanghai Engineering Center for Visual Science and Photomedicine, ${ }^{\dagger}$ Shanghai; and the Shanghai Key Laboratory of Fundus Diseases, ${ }^{\ddagger}$ Shanghai, People's Republic of China

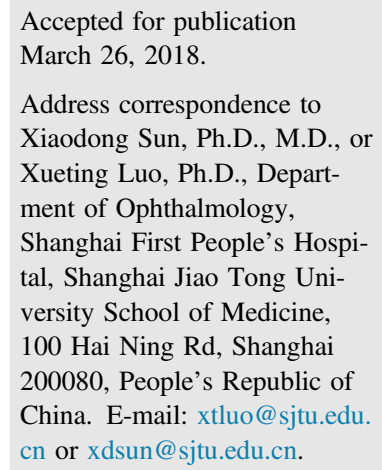

Photoreceptor degeneration is a leading cause of visual impairment worldwide. Separation of neurosensory retina from the underlying retinal pigment epithelium is a prominent feature preceding photoreceptor degeneration in a variety of retinal diseases. Although ophthalmic surgical procedures have been well developed to restore retinal structures, postoperative patients usually experience progressive photoreceptor degeneration and irreversible vision loss that is incurable at present. Previous studies point to a critical role of mitochondria-mediated apoptotic pathway in photoreceptor degeneration, but the upstream triggers remain largely unexplored. In this study, we show that after experimental retinal detachment induction, photoreceptors activate dynamin-related protein 1 (Drp1)-dependent mitochondrial fission pathway and subsequent apoptotic cascades. Mechanistically, endogenous reactive oxygen species (ROS) are necessary for Drp1 activation in vivo, and exogenous ROS insult is sufficient to activate Drp1-dependent mitochondrial fission in cultured photoreceptors. Accordingly, inhibition of Drp1 activity effectively preserves mitochondrial integrity and rescues photoreceptors. Collectively, our data delineate an ROS-Drp1-mitochondria axis that promotes photoreceptor degeneration in retinal diseased models. (Am J Pathol 2018, 188: 1713-1722; https://doi.org/10.1016/j.ajpath.2018.03.013)
Photoreceptors are light-sensing neurons responsible for visual signal reception. ${ }^{1}$ Because of sustained phototransduction and oxidative stress, ${ }^{2}$ photoreceptors are among the most metabolically active and energy-demanding tissues in the central nervous system. ${ }^{3}$ However, the outer retina, where photoreceptors reside in, mainly depends on retinal pigment epithelium layer and choroid vascular bed underneath for nutrition and oxygen to survive. ${ }^{4,5}$ Such unique organization makes photoreceptors exceptionally vulnerable to metabolic perturbations under stressed conditions, including rhegmatogenous retinal detachment, tractional diabetic retinopathy, and age-related macular degeneration. ${ }^{6,7}$ Clinically, palliative interventions remain the mainstay to halt disease progression, but in the long run, patients experience progressive vision loss because of irreversible photoreceptor death. ${ }^{8}$ Therefore, development of photoreceptor-targeted neuroprotective strategy is pivotal to preserve vision.

Photoreceptors degenerate mainly through apoptotic pathway. However, inhibition of death effectors does not effectively protect photoreceptors because of activation of necrotic pathway. ${ }^{9}$ Therefore, elucidation of mechanisms leading to photoreceptor degeneration, in particular identification of the initial triggers upstream of death effectors, is critical to protect photoreceptors. Nonetheless, the upstream signals of photoreceptor degeneration remain largely unexplored.

\footnotetext{
Supported by National Science Fund for Distinguished Young Scholars grant 81425006 (X.Su.), National Natural Science Foundation of China grant 81470640 (F.Wa.), Science and Technology Commission of Shanghai Municipality grants 16dz2251500 (F.Wa.) and 16140900800 (X.Luo), Shanghai Pujiang Program grant 16PJ1408500 (H.Z.), Program for Eastern Young Scholar at Shanghai Institutions of Higher Learning grant QD2016003 (X.Luo), Translational Medicine Innovation Fund of Shanghai Jiao Tong University School of Medicine grant 15ZH4005 (X.Su.), and Translational Medicine Innovation Fund of Shanghai Jiao Tong University School of Medicine grant 15ZH2009 (X.Su. and X.Lu).

$\mathrm{X}$. She and $\mathrm{X}$. Lu contributed equally to this work.

Disclosures: None declared.
} 
A

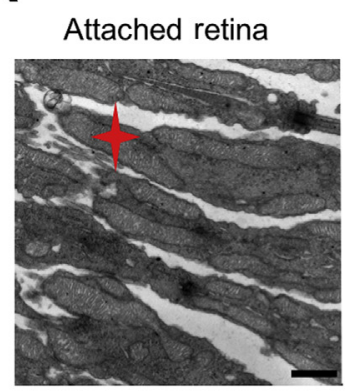

C

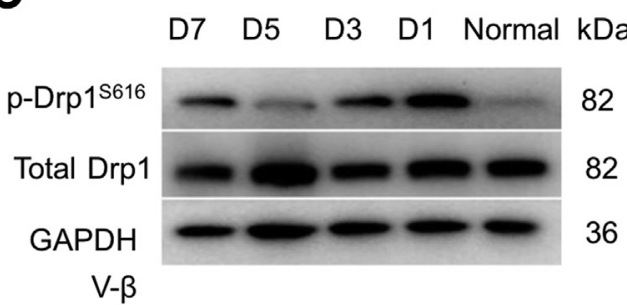

E
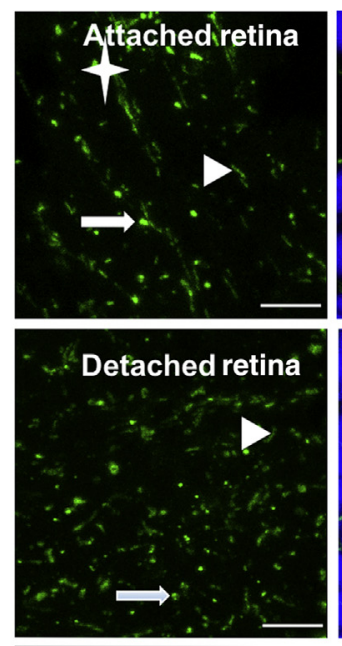

Detached retina

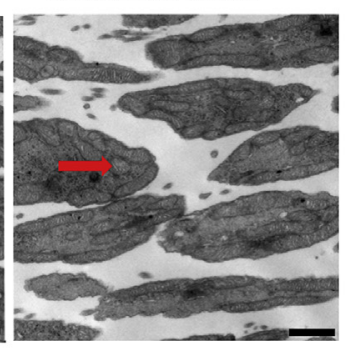

D

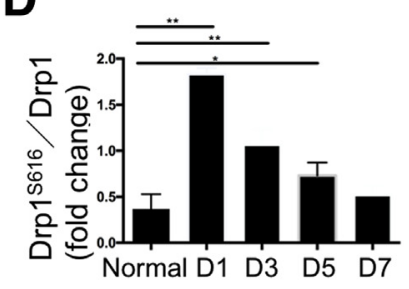

$\mathbf{F}$
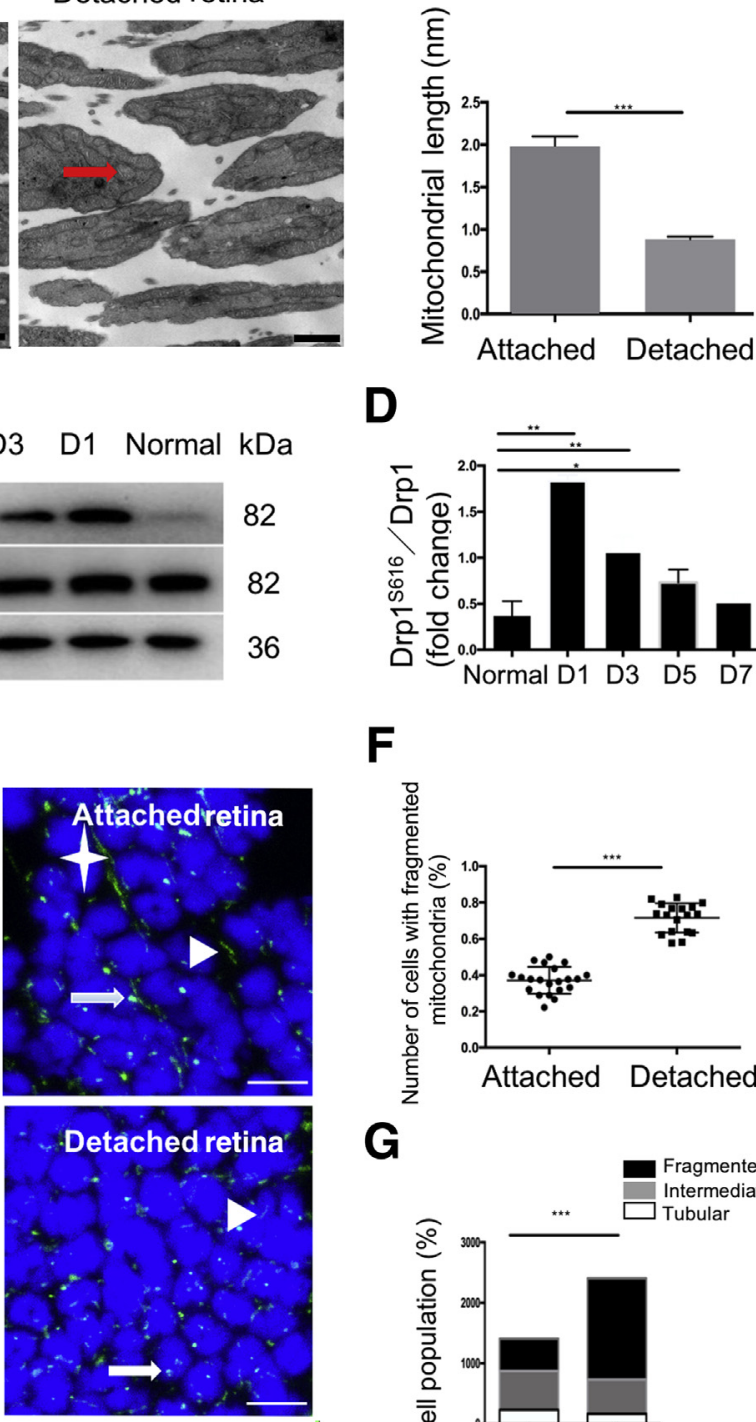

B

Figure 1 Mitochondrial dynamic changes in the detached retina. A: Representative transmission electron microscopy images show mitochondrial network of photoreceptors in attached retina and detached retina 3 days after retinal detachment (RD). Red arrow and star mark fragmented and tubular mitochondrial species, respectively. B: Quantification of mitochondrial length in photoreceptors; mitochondrial length become shorter after RD. C and D: Western blot analysis of retinal lysates after RD; quantification indicates that mitochondrial fission protein Drp $1^{\mathrm{S} 616}$ increases at 1,3 , and 5 days (D), and peaks 1 day, after RD. E: Mitochondrial network of the photoreceptors was labeled with Complex V (COX-V) $\beta$ subunit (V- $\beta$ ) antibodies and assessed by confocal microscopy in attached and detached retina 3 days after RD. Greater than half of mitochondria displaying the long tubular shape are tubular, less than half of mitochondria displaying the tubular shape are intermediate, and most mitochondria displaying short are fragmented (star, arrowhead, and arrow mark tubular, intermediate, and fragmented mitochondria, respectively). $\mathbf{F}$ and G: Quantification of mitochondrial morphology by ImageJ (cell number $=100$, mitochondria $=500$ ); the fragmentation ratio was defined as the percentage of fragmented mitochondria/total mitochondria. Fragmentation ratio and the distribution of fragmented mitochondrial morphology increase after RD. B, D, F, and G: Statistical analysis was performed via independent $t$-test (B), analysis of variance with Bonferroni coefficient (D), and $\chi^{2}$ test $(\mathbf{F}$ and $\mathbf{G})$, each with three independent experiments. Data are expressed as means \pm SD (B and $\mathbf{D})$ or as medians (F and $\mathbf{G}) . n=200(\mathbf{B}) ; n=3$ for each group (C and D). ${ }^{*} P<0.05,{ }^{* *} P<0.01$, and $* * * P<0.001$. Scale bars: $1 \mathrm{~nm}(\mathbf{A}) ; 50 \mu \mathrm{m}$ (E). GAPDH, glyceraldehyde-3-phosphate dehydrogenase.
Mitochondria are energy-producing organelles that play a central role in cell death decision. ${ }^{10}$ Accumulating evidence suggests that mitochondrial dysfunction precedes neuronal death in neurodegenerative diseases. ${ }^{11}$ More important, decline in mitochondrial metabolism is associated with age-related photoreceptor degeneration, ${ }^{12}$ indicating the importance of mitochondria for photoreceptor survival. To further clarify the role of mitochondria in photoreceptor degeneration, we used diseased models both in vivo and in vitro wherein photoreceptors were exposed to stress. Mitochondrial fission within photoreceptors represented an early event before activation of death effectors. At the molecular level, dynamin-related protein 1 (Drp1) was identified as the critical mediator of mitochondrial fission in stressed photoreceptors. Reactive oxygen species (ROS) were both required and sufficient to induce Drp1-dependent mitochondrial fission. Moreover, inhibition of Drp1 substantially preserved mitochondrial integrity and protected photoreceptors both structurally and functionally. Collectively, our findings have uncovered the critical role of mitochondria in photoreceptor degeneration and identified Drp1 as a promising therapeutic target for photoreceptor protection.

\section{Materials and Methods}

\section{Animals and Surgery}

The biomedical research was approved by the Shanghai General Hospital (Shanghai, China) review board. All procedures adhered to the Statement of the Association for Research in Vision and Ophthalmology for using animals in biomedical research. Sprague Dawley rats were housed at the Laboratory Animal Center of Shanghai General Hospital. Male 
A

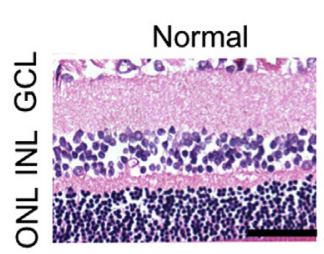

C

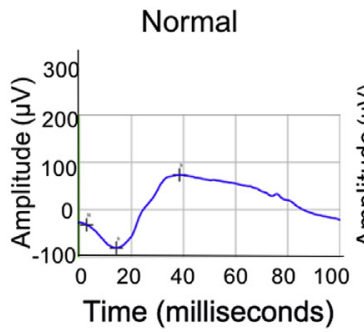

D

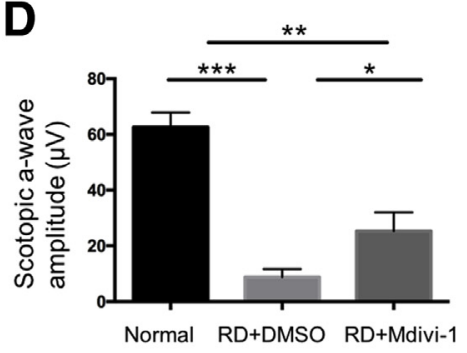

$\mathbf{F}$

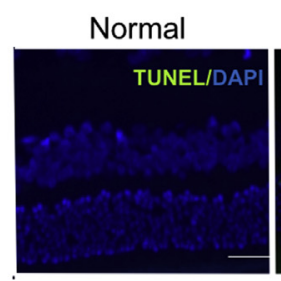

RD+DMSO

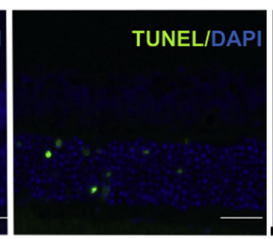

RD+DMSO

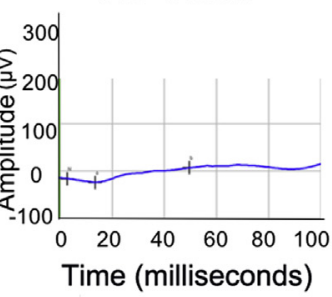

B

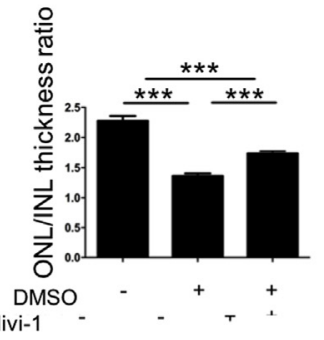
Mdivi-1
E

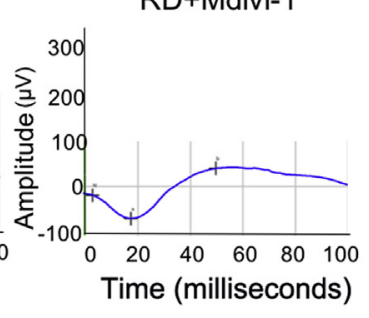

RD+Mdivi-1

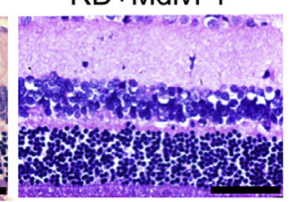

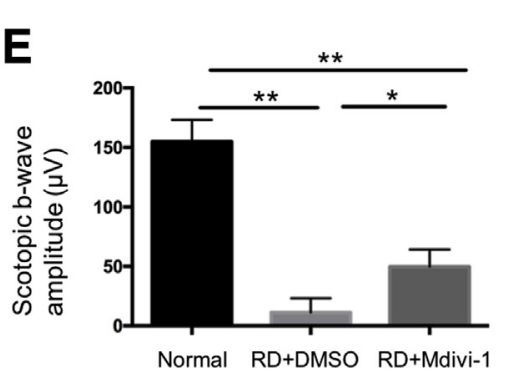

G

RD+Mdivi-1

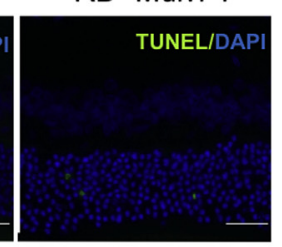

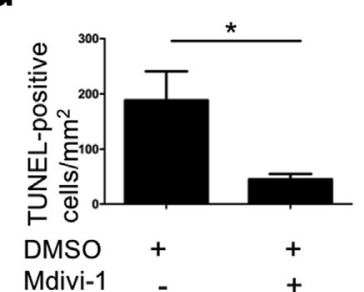

Figure 2 Inhibition of Drp1 attenuates retina function after retinal detachment. A and B: Representative hematoxylin and eosin-stained retinal sections show outer segment of the retina 7 days after retinal detachment (RD). The thickness of outer nuclear layer (ONL) normalized to that of inner nuclear layer (INL) was used as indication of photoreceptor survival. The ONL/INL thickness ratio in control [RD+ dimethyl sulfoxide (DMSO)] and study (RD+Mdivi-1) groups reduces after RD. C: Mdivi-1-treated group increases the functional recovery of scotopic electroretinography 7 days after RD. Representatives scotopic electroretinography wave forms from baseline, control, and study groups. D: Quantification of amplitude of scotopic a and b waves; the amplitude of a wave reduced in control and study groups after RD. E: The amplitude of $b$ wave reduced after RD. F and G: Quantification of terminal deoxynucleotidyl transferase-mediated dUTP nick-end labeling (TUNEL)-positive staining 3 days after RD in retina treated with vehicle and Mdivi-1. B, D, E, and G: Statistical analysis was performed via analysis of variance with Bonferroni coefficient $(\mathbf{B}, \mathbf{D}$, and $\mathbf{E}$ ) and an independent $t$-test $(\mathbf{G})$, all with three independent experiments. Data are expressed as means \pm SD (B, D, E, and $\mathbf{G}) . n=6(\mathbf{C}) ; n=5$ for each (F and $\mathbf{G}) .{ }^{*} P<0.05,{ }^{*} p<0.01$, and ${ }^{* * *} P<0.001$. Scale bar $=40 \mu \mathrm{m}(\mathbf{A}$ and $\mathbf{F})$. GCL, ganglion cell layer.

rats (age, 8 to 10 weeks; weight, 180 to $250 \mathrm{~g}$ ) were used to conduct retinal detachment, as previously described..$^{13}$ Briefly, the rats were anesthetized by i.p. injection with $1 \%$ sodium pentobarbital (Sigma-Aldrich, St. Louis, MO). Before surgery, pupils were dilated with $0.5 \%$ tropicamide and $0.5 \%$ phenylephrine hydrochloride eye drops (Santen Pharmaceutical Co. Ltd., Osaka, Japan). A needle head was used to generate a sclerotomy $2 \mathrm{~mm}$ posterior to the limbus. Then, a 30-gauge needle was introduced through the sclerotomy into the subretinal space. Sodium hyaluronate $(10 \mathrm{mg} / \mathrm{mL}$; LG Life Sciences, Jeollabuk-do, Korea) was injected to make two- thirds of the neurosensory retina detach from the underlying retinal pigment epithelium. The retinal detachment (RD) was verified by surgical microscope, and only the right eye was generated to RD, with left eye serving as normal control. All animal experiments included three to four rats per group and were repeated three times.

\section{Subretinal Injections of Mdivi-1 or N-Acetylcysteine}

Mdivi-1 (product number 338967-87-6; Sigma-Aldrich) was dissolved in $20 \%$ dimethyl sulfoxide and then further 

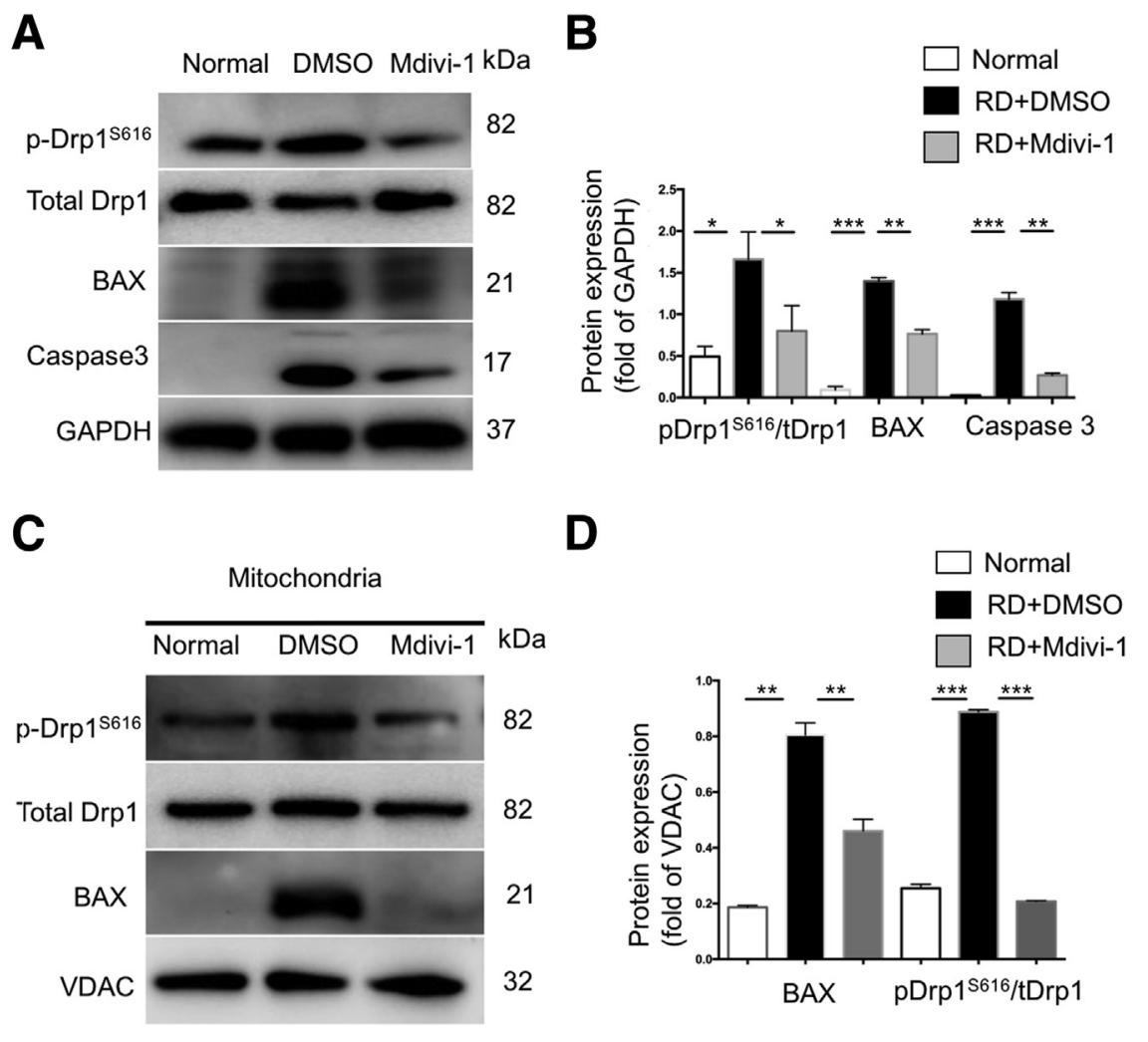

Figure 3 Inhibition of Drp1 protects the photoreceptors by suppressing intrinsic apoptosis way. A and B: Western blot analysis of the retinal lysates with Mdivi-1 treatment 3 days after retinal detachment (RD). Glyceraldehyde-3-phosphate dehydrogenase (GAPDH) was used as an internal control for analysis; Drp1 ${ }^{\text {S616 }}$ /total Drp1 (tDrp1), caspase3, and Bax increased after RD. C and D: Western blot analysis of the mitochondrial fraction with Mdivi-1 treatment 3 days after RD. Voltagedependent anion channel (VDAC) was used as a marker for mitochondria and as internal control. Quantification indicates that $\mathrm{p}$-Drp1 ${ }^{\mathrm{S} 616} / \mathrm{tDrp} 1$ and BAX increases after RD. B and D: Statistical analysis was performed via analysis of variance with Bonferroni coefficient, with three independent experiments. Data are expressed as means \pm SD (B and D). $n=3$ (A-D). ${ }^{*} P<0.05,{ }^{*} P<0.01$, and ${ }^{* * *} P<0.001$. DMS0, dimethyl sulfoxide. diluted with $0.9 \%$ physiological saline to a working concentration of $2.4 \mathrm{mg} / \mathrm{mL}$. N-acetylcysteine (product number A7250; Sigma-Aldrich) was dissolved in $0.9 \%$ physiological saline into the working concentration of 200 or $100 \mu \mathrm{mol} / \mathrm{L}$. Chemicals or vehicle $(5 \mu \mathrm{L})$ was injected into subretinal space at the time of RD induction.

\section{Cell Culture}

The $661 \mathrm{~W}$ cell line was maintained in Dulbecco's modified Eagle's medium containing $10 \%$ fetal bovine serum, $300 \mathrm{mg} / \mathrm{L}$ glutamine, $32 \mathrm{mg} / \mathrm{L}$ putrescine, $40 \mathrm{~mL} / \mathrm{L}$ of b-mercaptoethanol, and $40 \mathrm{mg} / \mathrm{L}$ of both hydrocortisone 21-hemisuccinate and progesterone. The medium also contained penicillin (90 U/mL) and streptomycin $(0.09 \mathrm{mg} / \mathrm{mL})$. Cells were maintained under regular cell culture chamber. $\mathrm{H}_{2} \mathrm{O}_{2}(500$ $\mu \mathrm{mol} / \mathrm{L}$ ) was used to mimic the oxidative stress-induced model for 24 hours. Mdivi-1 $(50 \mu \mathrm{mol} / \mathrm{L})$ was pretreated to 661 cells for 1 hour before $500 \mu \mathrm{mol} / \mathrm{L} \mathrm{H}_{2} \mathrm{O}_{2}$ treatment.

\section{Western Blot Analysis}

The retina or cultured cells were homogenized with lysis buffer containing $50 \mathrm{mmol} / \mathrm{L}$ Tris $7.4,150 \mathrm{mmol} / \mathrm{L} \mathrm{NaCl}, 1 \%$ Triton X-100, $1 \%$ sodium deoxycholate, $0.1 \%$ SDS, and inhibitors of protease (product number 11679498001; Roche, Mannheim, Germany) and phosphatase (product number P0044; Sigma-Aldrich). Samples were resolved by SDSPAGE and transferred to polyvinylidene difluoride membranes. Primary antibodies used for probing were listed below: phosphorylated Drp1(Ser616) (product number 3455; Cell Signaling Technology, Danvers, MA), Drp1 (product number 14647; Cell Signaling Technology), cleaved caspase3 (product number 25546-1-AP; Proteintech, Chicago, IL), Bax (product number ab32503; Abcam, Cambridge, UK), voltage-dependent anion channel (product number 4866; Cell Signaling Technology), glyceraldehyde-3-phosphate dehydrogenase (product number 60004; Proteintech), and $\beta$-actin (product number 4970; Cell Signaling Technology). After horseradish peroxidase-conjugated secondary antibodies and chemiluminescent substrates were applied, the signals were detected by Amersham Imager 600 (GE Medical Diagnostics Ltd., Little Chalfont, UK). Densitometry was measured and analyzed with ImageJ version $1.48(\mathrm{NIH}$, Bethesda, MD; http://imagej.nih.gov/ij).

\section{Mitochondrial Fission Confocal Microscopy}

The rats were perfused transcardially with $4 \%$ paraformaldehyde. The eyeballs were harvested, cryosectioned, and prepared for immunohistochemistry. $661 \mathrm{~W}$ cells were fixed with $4 \%$ paraformaldehyde before staining. Leica TCS SP8 confocal microscopy (Leica Microsystems, Weltzlar, Germany) was used to detect Complex V (COX-V) $\beta$ subunit antibody (product number ab14730; Abcam) labeled mitochondria with an excitation wavelength of $543 \mathrm{~nm}$ and an emission wavelength from 575 to $700 \mathrm{~nm}$. The image size was set to $1024 \times 1024$ pixels in a scan speed of $100 \mathrm{~Hz}$ with a $63 \times$ oil immersion lens. The way of 
A

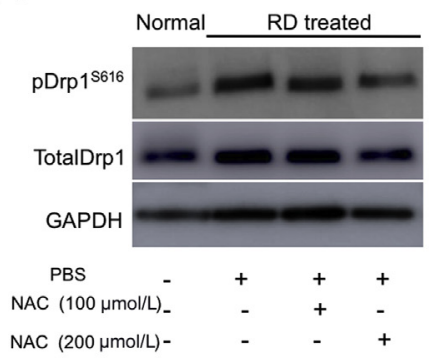

C

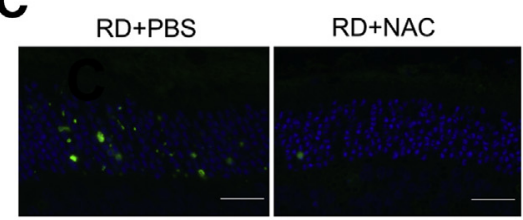

E

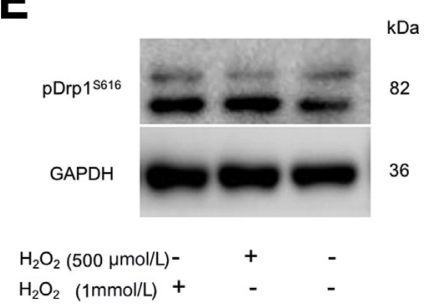

$\mathbf{G}_{\mathrm{V}-\beta}$

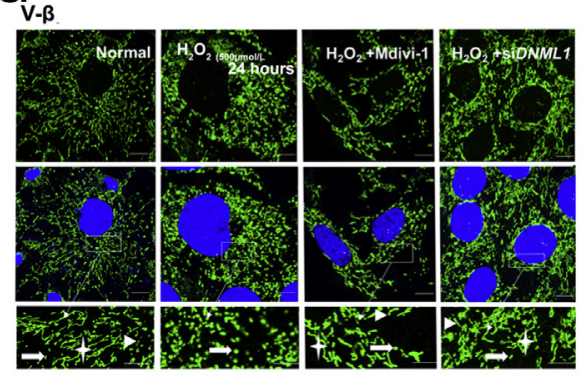

B

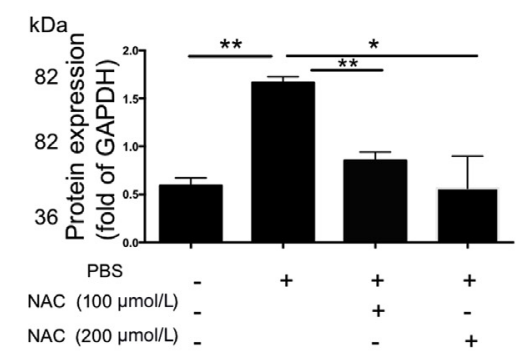

D

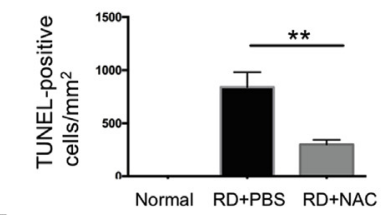

$\mathbf{F}$

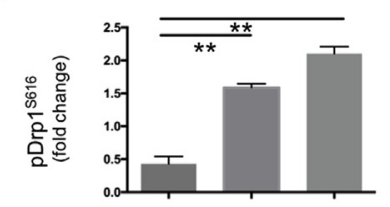

$\mathrm{H}_{2} \mathrm{O}_{2}(500 \mu \mathrm{mol} / \mathrm{L})-\quad+\quad-$ $\mathrm{H}_{2} \mathrm{O}_{2}(1 \mathrm{mmol} / \mathrm{L}) \quad-\quad-\quad+$

\section{H}

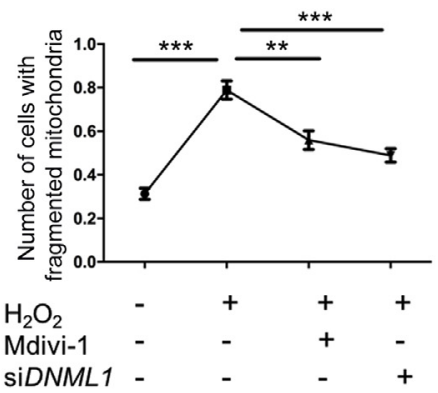

Figure 4 0xidative stress induced mitochondrial fission. $\mathbf{A}$ and $\mathbf{B}$ : Western blot analysis of $\mathrm{pDrps}^{616}$ /total Drp1 (tDrp1) in retinal lysates 3 days after retinal detachment (RD) and $\mathrm{N}$-acetylcysteine (NAC) treatment. Quantification results indicate that $\mathrm{pDrpS}^{616} / \mathrm{tDrp1}$ increases after RD; NAC (100 and $200 \mu \mathrm{mol} / \mathrm{L})$ treated group decreases the $\mathrm{pDrpS}^{616} / \mathrm{tDrp1}$ expression. Glyceraldehyde-3-phosphate dehydrogenase (GAPDH) was used as internal control. C and D: Representative terminal deoxynucleotidyl transferase-mediated dUTP nick-end labeling (TUNEL)-positive cells in photoreceptors with NAC $(200 \mu \mathrm{mol} / \mathrm{L})$ treatment 3 days after RD; quantification of TUNEL-positive results indicates that NAC treatment attenuates photoreceptor death. E and F: Representative Western blot analysis of pDrpS ${ }^{616}$ in different $\mathrm{H}_{2} \mathrm{O}_{2}$ concentrations insulted $661 \mathrm{~W}$ cells for 24 hours; quantification results indicate that $\mathrm{pDrpS}^{616} / \mathrm{tDrp1}$ increases after $\mathrm{H}_{2} \mathrm{O}_{2}$ treatment. GAPDH was used as internal control. $\mathbf{G}$ and $\mathbf{H}$ : A total of $500 \mu \mathrm{mol} / \mathrm{L}$ $\mathrm{H}_{2} \mathrm{O}_{2}$ was induced in $661 \mathrm{~W}$ cells for 24 hours; morphology of mitochondria was imaged by confocal microscopy and quantitated with ImageJ (cell $=100$, mitochondria $=500$ ). Boxed areas in middle panels in $\mathbf{G}$ are shown at higher magnification in the bottom panels. Star, arrowhead, and arrow mark tubular, intermediate, and fragmented mitochondria, respectively. Fragmented mitochondria increase after $\mathrm{H}_{2} \mathrm{O}_{2}$ treatment. B, D, F, and H: Statistical analysis was performed via analysis of variance with Bonferroni coefficient (B, D, and $\mathbf{F})$ and $\chi^{2}(\mathbf{H})$, all with three independent experiments. Data are expressed as means \pm SD (B, D, F, and $\mathbf{H}) . n=3$ (A and $\mathbf{B})$. ${ }^{*} P<0.05,{ }^{* *} P<0.01$, and ${ }^{* * *} P<0.001$. Scale bars: $40 \mu \mathrm{m}(\mathbf{C}) ; 10 \mu \mathrm{m}$ (G, top and middle panels); $5 \mu \mathrm{m}$ (G, bottom panels). Original magnification, $\times 6.0$ (G, bottom panels). PBS, phosphate-buffered saline. mitochondrial morphology was analyzed from Rehman et al. ${ }^{14,15}$ A total of 10 to 15 cells were captured randomly, and the images were analyzed using ImageJ. Greater than half of mitochondria displaying the long tubular shape are tubular, less than half of mitochondria displaying the long tubular shape are intermediate, and most mitochondria displaying short are fragmented shape. ${ }^{15}$ Fragmentation ratio is defined as number of the fragmented mitochondria in total cells. One hundred cells were statistically analyzed by a blind observer (X.Lu). ${ }^{16}$

\section{Histopathological Retinal ONL Damage Assessment}

Eyes were enucleated 7 days after retinal detachment. Each group consisted of three eyes each time and repeated three times. Sections ( $5 \mu \mathrm{m}$ thick) were in the vertical meridian and inferior portion of the eye wall and stained with hematoxylin and eosin. Sections in the angle were excluded in our research, because the retinal thickness varies with the distance from the optic nerve; the inner nuclear layer thickness was used as the internal control, at the same distance from the optic nerve. ${ }^{17}$ Five points in each section were measured by skilled observer (X.Sh.), and five sections were randomly selected in each eye. The thickness ratio of the outer nuclear layer (ONL)/inner nuclear layer was calculated to compare the ONL damage in each group. ${ }^{18,19}$

\section{Transmission Electron Microscopy}

Transmission electron microscopy analysis was performed, as described previously. ${ }^{20}$ Briefly, the retina was fixed in a solution containing $5 \%$ formaldehyde and $2 \%$ glutaraldehyde in 0.1 $\mathrm{mol} / \mathrm{L}$ phosphate-buffered saline, $\mathrm{pH}$ 7.4. The fixed and processed samples were subjected to imaging with transmission electron microscopy (Leica TCS SP8; Leica, Buffalo Grove, IL). A total of 200 mitochondria of photoreceptor from three 
A

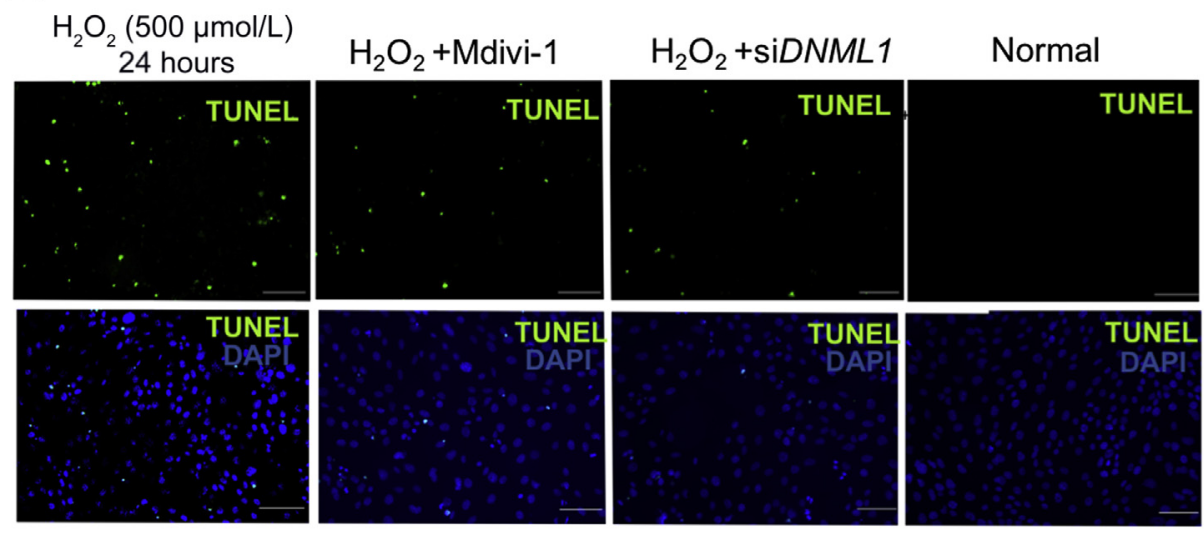

B

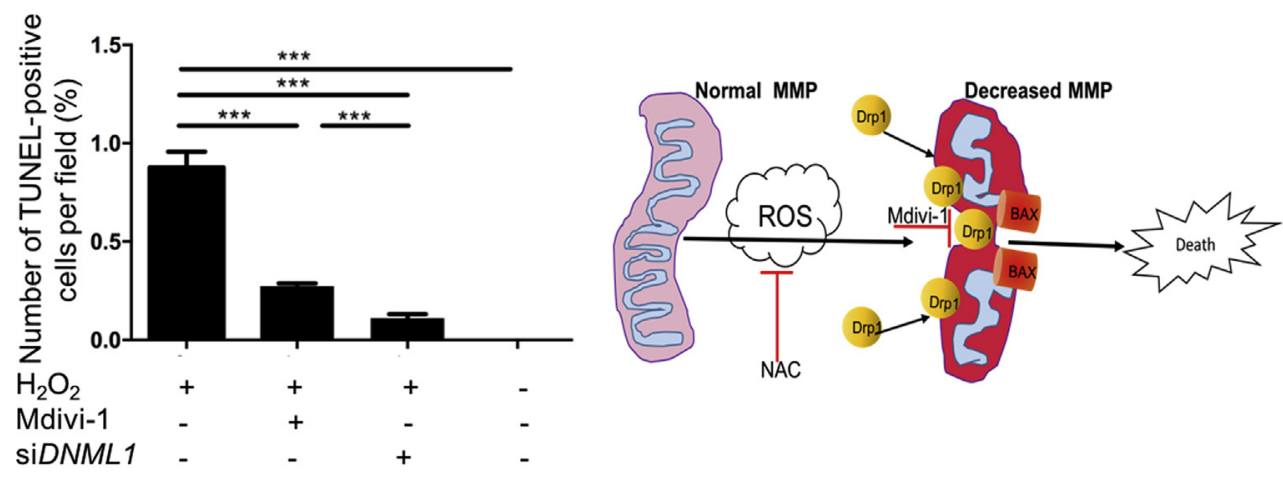

D
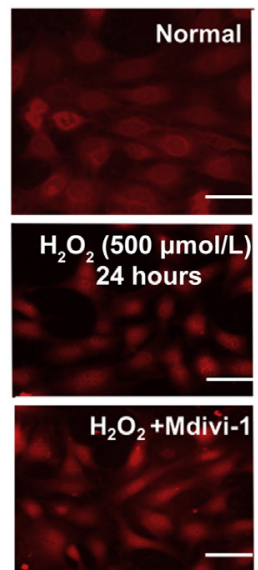
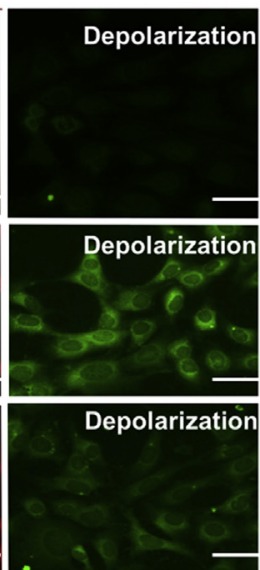
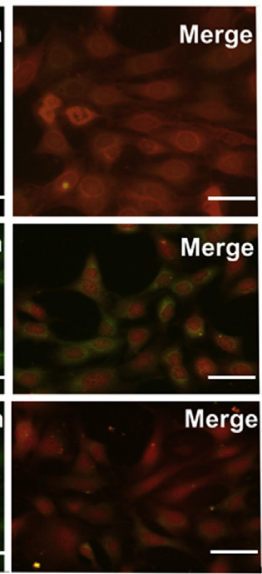

$\mathbf{E}$

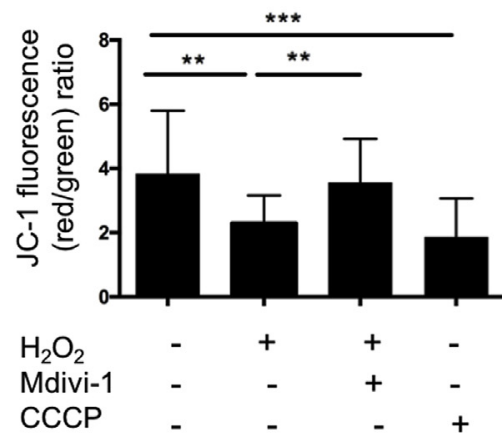

Figure 5 Inhibition of Drp1 suppresses apoptosis and protects $661 \mathrm{~W}$ cells through protecting mitochondrial integrity after $\mathrm{H}_{2} \mathrm{O}_{2}$ insult. A: Representative terminal deoxynucleotidyl transferase-mediated dUTP nick-end labeling (TUNEL) staining after treatment; either Mdivi-1 or siDNML1 is effective in rescuing $661 \mathrm{~W}$ cells after $500 \mu \mathrm{mol} / \mathrm{L} \mathrm{H}_{2} \mathrm{O}_{2}$ insult for 24 hours. B: Quantification of TUNEL-positive cells indicates that $\mathrm{H}_{2} \mathrm{O}_{2}$ treatment induces cell death. C: Diagram shows that reactive oxygen species (ROS) - Drp1-dependent mitochondrial fission joins in the photoreceptors' death after retinal detachment (RD). The increasing ROS is an important upstream step for Drp1 activation. Drp1-dependent mitochondrial fission is companied with BAX activation after RD; inhibition of Drp1 with Mdivi-1 could suppress BAX activation and maintain mitochondrial integrity. D: Representative mitochondrial membrane potential (MMP) by JC-1 staining in different groups. JC-1 dye expresses red signals with high membrane potential and shifts to green fluorescence when mitochondria are decoupled. The ratio of green/red intensity was measured as the depolarization of mitochondrial membrane potential. E: Quantification of red and green fluorescence intensity and the red/green ratio after JC-1 staining in the $\mathrm{H}_{2} \mathrm{O}_{2}$-induced model for 24 hours. The ratio decreases after $\mathrm{H}_{2} \mathrm{O}_{2}$ and $C C C P$ treatment, whereas Mdivi- 1 rescues $\mathrm{H}_{2} \mathrm{O}_{2}$-induced loss of mitochondrial membrane potential and increases the red/green ratio. Statistical analysis was performed via analysis of variance with Bonferroni coefficient, with three independent experiments. Data are expressed as means \pm SD (B and $\mathbf{E}) .{ }^{*} P<0.01,{ }^{* \star \star} P<0.001$. Scale bar $=40 \mu \mathrm{m}(\mathbf{A}$ and $\mathbf{D})$. NAC, N-acetylcysteine. 
different animals were digitized, and the mitochondrial length was measured with the use of ImageJ for analysis.

\section{Mitochondrial Isolation}

Mitochondrial isolation from retina was performed using a mitochondrial isolation kit (product number C3606; Beyotime, Shanghai, China), according to the instructions. Briefly, dissected retina was homogenized in cold mitochondrial lysis buffer. The homogenate was then centrifuged at $600 \times g$ for 10 minutes at $4^{\circ} \mathrm{C}$ to spin down the nuclei and unbroken cells. The supernatant was collected and centrifuged again at $12,000 \times g$ for 30 minutes at $4{ }^{\circ} \mathrm{C}$ to collect the mitochondria in the supernatant.

\section{Apoptosis Assay}

DNA fragmentation was detected using terminal deoxynucleotidyl transferase-mediated dUTP nick-end labeling (TUNEL; In Situ Cell Death Detection Kit; product number 11684795910; Roche Diagnostics, Meylan, France) 24 hours after hydrogen peroxide treatment. TUNEL-positive staining was accounted in four random fields for each group and repeated three times. To detect the photoreceptor death in ONL, TUNEL staining was performed according to the manufacturer's instructions. Five sections per eye were selected, and the TUNEL-positive cells in the ONL were counted by adjusting to the numbers of cells per $1 \mathrm{~mm}^{2}$.

\section{RNA Interference and Transfection}

Small double-stranded RNAs (siRNAs) for Dynamin 1 like (DNML1) were designed, and negative control sequences were purchased from GenePharma Inc. (Shanghai, China). 661W cells were transfected with siRNA/lipofectamine mixture, according to the manufacturer's instructions. Four pair of siRNAs were designed and tested, with the most effective one selected in this study $^{21}$ : siDNML1, 5'-AGGAGAAGAAAAUGGUAAAUUUCTT- $3^{\prime}$ (sense) and $5^{\prime}$-GAAAUUUACCAUUUUCUUCUCCUTT- $3^{\prime}$ (antisense); and negative control, 5'-UUCUCCGAACGUGUCACGUTT-3' (sense) and $5^{\prime}$-ACGUGACACGUUCGGAGAATT- $3^{\prime}$ (antisense). The final concentration of siRNA was $20 \mathrm{pmol} / \mathrm{L}$ per well. The medium was changed 24 hours after transfection. Cells were harvested 48 hours after transfection. Efficiency of knocking down endogenous mouse $D N M L 1$ was verified by Western blot analysis, and the transfection efficiency is calculated by the expression of p-Drp $1^{\text {Ser616 }}$ between siDNML1 and control group relative to $\beta$-actin.

\section{Mitochondrial Membrane Potential Assay}

Dual-emission potential-sensitive probe JC-1 (product number T3168; Molecular Probes, Eugene, OR) was used to measure the mitochondrial membrane potential. $661 \mathrm{~W}$ cells were washed with cold phosphate-buffered saline after treatment and then incubated with $10 \mu \mathrm{L}$ of JC- 1 for 20 minutes at $37^{\circ} \mathrm{C}$, according to manufacturer's instructions. After incubation, cells were washed twice by cold phosphate-buffered saline; the red fluorescence was measured with excitation at $525 \mathrm{~nm}$ and emission at 590 $\mathrm{nm}$, and the green fluorescence was measured with excitation at $490 \mathrm{~nm}$ and emission at $530 \mathrm{~nm}$, in a random order and analyzed with ImageJ. The ratio of red/green fluorescence was used to reflect mitochondrial membrane potential.

\section{Cell Viability Assay}

The effect of hydrogen peroxide on the $661 \mathrm{~W}$ cells was measured by using the cell counting kit-8 (Sigma-Aldrich). The cells were plated onto a 96-well culture at a density of $1 \times 10^{4}$ cells per well and underwent different hydrogen peroxide treatments. Then, cell counting kit- 8 dye $(10 \mu \mathrm{L})$ was added to each well. After 4 hours, cells were incubated at $37^{\circ} \mathrm{C}$. The absorbance at $450 \mathrm{~nm}$ of each well was measured using a multifunction microplate reader (Molecular Devices, Sunnyvale, CA). All experiments were repeated three times.

\section{Electroretinography}

The retinal function was assessed by electroretinography before and 7 days after retinal detachment. The rats were pretreated overnight for dark adaptation and anesthetized, and the pupils were dilated, as previously introduced. The body temperature was set to $37^{\circ} \mathrm{C}$ with a heating pad during the procedure, a gold wire electrode was on the cornea, a reference electrode was at the head, and a ground electrode was in the tail. All of the procedures were performed in dim red light. The response to a light flash (3.0 candela seconds $/ \mathrm{m}^{2}$ ) from a photic stimulation was amplified; the preamplifier bandwidth was set at 0.2 to 300 $\mathrm{Hz}$. The a-wave amplitude was from baseline to the maximum a-wave. The amplitude of $b$-wave was measured from the maximum a-wave to the maximum b-wave peak. The amplitudes of $a$ and $b$ waves were used to evaluate the retinal function.

\section{Statistical Analysis}

Data were shown as means \pm SD or medians. The differences in two groups were analyzed by unpaired $t$-test. The mitochondrial length and electroretinography data in three or more groups were analyzed by one-way analysis of variance with Bonferroni coefficient. Mitochondrial morphologic features were analyzed by $\chi^{2}$ test by SPSS software version 21.0 for Windows (SPSS Inc., Chicago, IL). $P<0.05$ was considered as significant. 


\section{Results}

\section{Mitochondrial Fission Is Evident in the Photoreceptors} after Experimental RD

Mitochondria, structurally dynamic organelles, undergo constant fusion and fission to regulate their homeostasis. ${ }^{22}$ Increased mitochondrial fission is associated with functional deficiency under diseased conditions. ${ }^{23}$ To directly visualize mitochondria morphology in vivo, transmission electron microscopy was used to analyze the retinal tissues of rats on the third day after experimental $\mathrm{RD}$. The mitochondria within photoreceptors appeared to be fragmented, punctate, and scattered compared with the typically long and interconnected structures in untreated control (Figure 1, A and B). The mitochondria were also labeled with antibodies against $\mathrm{COX}-\mathrm{V} \beta$ subunit for analysis of mitochondrial morphology with confocal microscopy. In normal photoreceptors, rod-like structures in various lengths were readily detectable. However, punctate and dispersed mitochondrial signals dominated the diseased photoreceptors after RD (Figure 1, E-G). The mitochondria were artificially divided into three species (ie, fragmented, intermediate, and tubular) on the basis of their morphology, and the distribution of mitochondrial structures was further assessed. ${ }^{24}$ Consistent with the results of transmission electron microscopy analysis, the amount and proportion of fragmented mitochondrial species increased substantially with concurrent decrease of intermediate and tubular species moderately in photoreceptors after RD (Figure 1, F and G).

\section{Drp1 Activation Is Critical to Mitochondrial Fission and Subsequent Photoreceptor Death}

Drp1 is the essential mediator of mitochondrial fission, and its phosphorylation is associated with activation of the mitochondrial fission pathway. ${ }^{25}$ To assess whether Drp1 is involved in the process of mitochondrial fission in photoreceptors after RD, the retina was dissected out for analysis by Western blot. Drp1 appeared to be phosphorylated abruptly after injury (Figure 1, C and D). The p-Drp1 signal peaked within 1 day after $\mathrm{RD}$, followed by gradual decline, suggesting a potential role of Drp1 in RD-induced mitochondrial fission in photoreceptors. Thus, RD induced Drp1 activation and mitochondrial fission in the photoreceptors.

\section{Inhibition of Drp1 Attenuates Mitochondrial Fission and Photoreceptor Degeneration after RD}

Apoptosis is the primary pathway through which photoreceptors degenerate. ${ }^{9}$ Consistently, significant up-regulation of TUNEL staining was detected at 3 days after RD (Figure 2, F and G). Because activation of Drp1 preceded TUNEL staining (Figures $1 \mathrm{C}$ and $2 \mathrm{~F}$ ), we imply that Drp1mediated mitochondrial fission may serve as upstream signals regulating the apoptotic pathway in photoreceptors after RD. To further verify this hypothesis, Mdivi-1, a highly selective inhibitor for Drp $1,{ }^{26}$ was subretinally administered at the time of RD induction. As expected, treatment with Mdivi-1 attenuated photoreceptor death (Figure 2, F and G). The therapeutic potential of Mdivi-1 in experimental RD model was further assessed. After retinal detachment, the progressive degeneration of photoreceptors results in a gradual thinning of the ONL. Therefore, the thickness of ONL serves as an indicator of photoreceptor survival. RD induced substantial loss of photoreceptors, whereas Mdivi-1 treatment effectively preserved the ONL structure in the retina (Figure 2, A and B). Consistently, the retinal function was significantly rescued with Mdivi-1 treatment, as examined by scotopic electroretinography (Figure 2, C-E).

Next, the activity of apoptotic factors was analyzed in the retinal tissues. The expression of cleaved caspase-3 was substantially suppressed by Mdivi-1 after RD (Figure 3, A and B). The Bcl-2 family is a critical regulator of mitochondrial permeability and intrinsic apoptotic pathway. ${ }^{27}$ Previous report has demonstrated a critical role of Bax, a member of the Bcl-2 family, in photoreceptor degeneration after $\mathrm{RD}{ }^{28}$ To test whether Bax is involved in Mdivi-1-mediated suppression of caspase-3 activity, the expression level of Bax was further analyzed in the retinal lysates after RD. Bax expression was upregulated significantly after $\mathrm{RD}$, whereas Mdivi-1 treatment substantially attenuated Bax activity (Figure 3, A and B). Both Drp1 and Bax are cytoplasmic proteins that translocate onto mitochondria membrane on activation. ${ }^{29-31}$ To this end, the mitochondrial fraction was extracted from the retinal lysate, and it was found that both activated Drp1 and Bax protein in mitochondria were substantially attenuated (Figure 3, C and D).

Taken together, we conclude that Drp1 inhibition by Mdivi-1 has a neuroprotective impact on the retina in experimental RD model by suppressing mitochondrial fission and the apoptotic pathway.

\section{Drp1-Mediated Mitochondrial Fission Is Induced by Oxidative Stress}

Oxidative stress has been well documented as a danger signal to photoreceptors. Accordingly, alleviation of oxidative stress protects photoreceptors after RD. ${ }^{32}$ Previous studies indicated potential correlations between oxidative stress and mitochondrial fission. ${ }^{33}$ However, their causal relationship remains to be explored. Therefore, it was questioned whether attenuation of oxidative stress would have an effect on mitochondrial dynamics in photoreceptors after RD. N-acetylcysteine, a welldefined scavenger of ROS, was introduced subretinally at the time of RD induction, and the expression level of activated Drp1 was examined. $\mathrm{N}$-acetylcysteine effectively suppressed the expression of p-Drp1 and attenuated photoreceptor death after $\mathrm{N}$-acetylcysteine treatment (Figure 4, A-D), indicating a positive role of oxidative stress in promoting mitochondrial fission.

To further elucidate the causal relationship between oxidative stress and mitochondrial fission, an in vitro model of cultured murine photoreceptor-derived $661 \mathrm{~W}$ cell line challenged by hydrogen peroxide was used. Consistent with 
findings in vivo, hydrogen peroxide insult activated Drp1 (Figure 4, E and F). Immunostaining of $661 \mathrm{~W}$ cells with mitochondria-specific COX-V $\beta$ subunit antibodies revealed predominance of fragmented mitochondrial signals after hydrogen peroxide insult (Figure 4, G and $\mathrm{H}$ ), which is in agreement with the in vivo findings (Figure 4A). Consistently, DNML1 knockdown by siRNA (Supplemental Figure S1, A and B) also preserved mitochondrial integrity (Figure 4, G and $\mathrm{H})$, confirming the critical role of Drp1.

\section{Drp1 Inhibition Suppresses 0xidative Stress-Induced Degenerative Signals}

Hydrogen peroxide insult up-regulated intracellular ROS level of cultured $661 \mathrm{~W}$ cells and cell degeneration, whereas inhibition of Drp1 activity by Mdivi-1 or siRNAs effectively suppressed hydrogen peroxide-induced TUNEL activity (Figure 5, A and B). Pretreatment of $661 \mathrm{~W}$ cells with Mdivi-1 effectively preserved cell viability (Supplemental Figure S2) and mitochondrial membrane potential (Figure 5, D and E), which supports a role of Drp1 in oxidative stress-induced mitochondrial dysfunction. These findings strongly suggest that Drp1 and Drp1-dependent pathways may join in preserving the mitochondrial function. Oxidative stressinduced photoreceptor degeneration is at least in part mediated by Drp1-dependent mitochondrial fission.

\section{Discussion}

Photoreceptors play pivotal roles in visual reception by several energy-demanding activities, including phototransduction, resolution of light and oxygen-induced damage, and synthesis and replenishment of disk membranes. ${ }^{2}$ Therefore, photoreceptors necessitate sustained supply of oxygen and nutrition from the retinal pigment epithelium/ choroid complex to support highly active metabolism. ${ }^{4,5}$ Accordingly, photoreceptors hold a large reservoir of mitochondria to satisfy their metabolic demand. ${ }^{34}$ It has been reported that increased mitochondrial fission is associated with photoreceptor degeneration in aged mice. ${ }^{12}$ Moreover, mitochondrial abnormality correlates with photoreceptor degeneration in acute glucose deprivation model. ${ }^{35}$ However, the relationship between mitochondrial abnormality and photoreceptor death remains unknown.

In the case of $\mathrm{RD}$, as manifested in multiple retinal diseases, photoreceptors experience excessive ROS that are considered to trigger cell death, but the exact mechanisms remain to be elucidated. Accumulating evidence suggests that mitochondria are the major source of intracellular ROS in response to cellular stress. ${ }^{36}$ Consistently, a predominantly fissured population of mitochondria was observed in the photoreceptors of rats after experimental $\mathrm{RD}$, which represents an early degenerative event (Figure 1, C and D, and Figure 2, F and G). Because fissured mitochondria have been associated with energetic failure and cell degeneration, ${ }^{37}$ we speculate that mitochondrial fission could be the milestone along the ROS-induced degenerative pathway determining photoreceptor death. At the molecular level, Drp1, the key mediator of mitochondrial fission, was activated after RD. Notably, inhibition of ROS suppressed Drp1 activation, which supports a regulatory role of ROS in Drp1 activity. In vitro, hydrogen peroxide induced Drp1 activation, mitochondrial fission, and photoreceptor death, whereas inhibition of Drp1 preserved mitochondrial integrity and protected photoreceptors. Collectively, these data suggest that Drp1dependent mitochondrial fission plays key roles in mediating RD-induced photoreceptor degeneration.

It is intriguing to target mitochondria for photoreceptor protection because mitochondria are considered to be the major organelles that sense cellular stress and broadcast deathpromoting signals. Mitochondrial fission may represent a common pathway through which photoreceptors degenerate in other retinal diseased models. In conclusion, these findings suggest that Drp1-dependent mitochondrial fission plays critical roles in photoreceptor degeneration and that Drp1 inhibition is effective in preserving photoreceptors. Thus, Drp1 represents a potential therapeutic target for photoreceptor protection.

\section{Acknowledgments}

We thank Dr. Yu Chen (Yueyang Hospital, Shanghai University of Traditional Chinese Medicine) for providing the 661W photoreceptor cell line.

X.Sh., F.Wa., X.Luo, and X.Su. designed the study, analyzed data, and wrote the manuscript; X.Sh., X.Luo, T.L., X.Lu, J.S., Y.Z., S.Y., Q.G., F.We., and J.L. performed experiments; and H.Z. and X.Lu designed the study and analyzed data.

\section{Supplemental Data}

Supplemental material for this article can be found at https://doi.org/10.1016/j.ajpath.2018.03.013.

\section{References}

1. Nakanishi S: Second-order neurons and receptor mechanisms in visualinformation and olfactory-information processing. Trends Neurosci $1995,18: 359-364$

2. Ahmed J, Braun RD, Dunn R, Linsenmeier RA: Oxygen distribution in the macaque retina. Invest Ophthalmol Vis Sci 1993, 34:516-521

3. Okawa H, Sampath AP, Laughlin SB, Fain GL: ATP consumption by mammalian rod photoreceptors in darkness and in light. Curr Biol 2008, 18:1917-1921

4. Linsenmeier RA, Padnick-Silver L: Metabolic dependence of photoreceptors on the choroid in the normal and detached retina. Invest Ophthalmol Vis Sci 2000, 41:3117-3123

5. Luo L, Uehara H, Zhang X, Das SK, Olsen T, Holt D, Simonis JM, Jackman K, Singh N, Miya TR, Huang W, Ahmed F, BastosCarvalho A, Le YZ, Mamalis C, Chiodo VA, Hauswirth WW, Baffi J, Lacal PM, Orecchia A, Ferrara N, Gao G, Young-Hee K, Fu Y, Owen L, Albuquerque R, Baehr W, Thomas K, Li DY, Chalam KV, Shibuya M, Grisanti S, Wilson DJ, Ambati J, Ambati BK: 
Photoreceptor avascular privilege is shielded by soluble VEGF receptor-1. ELife 2013, 2:e00324

6. Arroyo JG, Yang L, Bula D, Chen DF: Photoreceptor apoptosis in human retinal detachment. Am J Ophthalmol 2005, 139:605-610

7. Lim LS, Mitchell P, Seddon JM, Holz FG, Wong TY: Age-related macular degeneration. Lancet 2012, 379:1728-1738

8. Wubben TJ, Besirli CG, Zacks DN: Pharmacotherapies for retinal detachment. Ophthalmology 2016, 123:1553-1562

9. Murakami Y, Notomi S, Hisatomi T, Nakazawa T, Ishibashi T, Miller JW, Vavvas DG: Photoreceptor cell death and rescue in retinal detachment and degenerations. Prog Retin Eye Res 2013, 37:114-140

10. Vakifahmetoglu-Norberg H, Ouchida AT, Norberg E: The role of mitochondria in metabolism and cell death. Biochem Biophys Res Commun 2017, 482:426-431

11. Ishihara N, Nomura M, Jofuku A, Kato H, Suzuki SO, Masuda K, Otera H, Nakanishi Y, Nonaka I, Goto Y, Taguchi N, Morinaga H, Maeda M, Takayanagi R, Yokota S, Mihara K: Mitochondrial fission factor Drp1 is essential for embryonic development and synapse formation in mice. Nat Cell Biol 2009, 11:958-966

12. Kam JH, Jeffery G, Hoh Kam J, Jeffery G: To unite or divide: mitochondrial dynamics in the murine outer retina that preceded age related photoreceptor loss. Oncotarget 2015, 6:26690-26701

13. Liu H, Qian J, Wang F, Sun X, Xu X, Xu W, Zhang X, Zhang X: Expression of two endoplasmic reticulum stress markers, GRP78 and GADD153, in rat retinal detachment model and its implication. Eye (Lond) 2009, 24:137-144

14. Rehman J, Zhang HJ, Toth PT, Zhang Y, Marsboom G, Hong Z, Salgia R, Husain AN, Wietholt C, Archer SL: Inhibition of mitochondrial fission prevents cell cycle progression in lung cancer. FASEB J 2012, 26:2175-2186

15. Wang L, Yu T, Lee H, O'Brien DK, Sesaki H, Yoon Y: Decreasing mitochondrial fission diminishes vascular smooth muscle cell migration and ameliorates intimal hyperplasia. Cardiovasc Res 2015, 106: 272-283

16. Ballweg K, Mutze K, Königshoff M, Eickelberg O, Meiners S: Cigarette smoke extract affects mitochondrial function in alveolar epithelial cells. Am J Physiol Lung Cell Mol Physiol 2014, 307:L895-L907

17. Dong K, Zhu H, Song Z, Gong Y, Wang F, Wang W, Zheng Z, Yu Z, Gu Q, Xu X, Sun X: Necrostatin-1 protects photoreceptors from cell death and improves functional outcome after experimental retinal detachment. Am J Pathol 2012, 181:1634-1641

18. Besirli CG, Chinskey ND, Zheng QD, Zacks DN: Inhibition of retinal detachment-induced apoptosis in photoreceptors by a small peptide inhibitor of the fas receptor. Invest Ophthalmol Vis Sci 2010, 51:2177-2184

19. Trichonas G, Murakami Y, Thanos A, Morizane Y, Kayama M, Debouck CM, Hisatomi T, Miller JW, Vavvas DG: Receptor interacting protein kinases mediate retinal detachment-induced photoreceptor necrosis and compensate for inhibition of apoptosis. Proc Nati Acad Sci U S A 2010, 107:21695-21700

20. Huang Q, Li J, Xing J, Li W, Li H, Ke X, Zhang J, Ren T, Shang Y, Yang H, Jiang J, Chen Z: CD147 promotes reprogramming of glucose metabolism and cell proliferation in HCC cells by inhibiting the p53dependent signaling pathway. J Hepatol 2014, 61:859-866

21. Wei JX, Yang J, Sun JF, Jia LT, Zhang Y, Zhang HZ, Li X, Meng YL, Yao LB, Yang AG: Both strands of siRNA have potential to guide posttranscriptional gene silencing in mammalian cells. PLoS One 2009, 4:e5382

22. Westermann B: Mitochondrial fusion and fission in cell life and death Nat Rev Mol Cell Biol 2010, 11:872-884

23. Archer SL: Mitochondrial dynamics: mitochondrial fission and fusion in human diseases. N Engl J Med 2013, 369:2236-2251

24. Picard M, Shirihai OS, Gentil BJ, Burelle Y: Mitochondrial morphology transitions and functions: implications for retrograde signaling? Am J Physiol Integr Comp Physiol 2013, 304:R393-R406

25. Kashatus JA, Nascimento A, Myers LJ, Sher A, Byrne FL, Hoehn KL, Counter CM, Kashatus DF: Erk2 phosphorylation of Drp1 promotes mitochondrial fission and MAPK-driven tumor growth. Mol Cell 2015, $57: 537-552$

26. Tanaka A, Youle RJ: A chemical inhibitor of DRP1 uncouples mitochondrial fission and apoptosis. Mol Cell 2008, 29:409-410

27. Chipuk JE, Moldoveanu T, Llambi F, Parsons MJ, Green DR: The BCL-2 family reunion. Mole Cell 2010, 37:299-310

28. Yang L, Bula D, Arroyo JG, Chen DF: Preventing retinal detachmentassociated photoreceptor cell loss in bax-deficient mice. Invest Ophthalmol Vis Sci 2004, 45:648-654

29. Cassidy-Stone A, Chipuk JE, Ingerman E, Song C, Yoo C, Kuwana T, Kurth MJ, Shaw JT, Hinshaw JE, Green DR, Nunnari J: Chemical inhibition of the mitochondrial division dynamin reveals its role in Bax/Bak-dependent mitochondrial outer membrane permeabilization. Deve Cell 2008, 14:193-204

30. Frank S, Gaume B, Bergmann-Leitner ES, Leitner WW, Robert EG, Catez F, Smith CL, Youle RJ: The role of dynamin-related protein 1, a mediator of mitochondrial fission, in apoptosis. Dev Cell 2001, 1: $515-525$

31. Große L, Wurm CA, Brüser C, Neumann D, Jans DC, Jakobs S: Bax assembles into large ring-like structures remodeling the mitochondrial outer membrane in apoptosis. EMBO J 2016, 35:402-413

32. Roh MI, Murakami Y, Thanos A, Vavvas DG, Miller JW: Edaravone, an ROS scavenger, ameliorates photoreceptor cell death after experimental retinal detachment. Invest Ophthalmol Vis Sci 2011, 52:3825-3831

33. Yu T, Wang L, Lee H, O'Brien DK, Bronk SF, Gores GJ, Yoon Y: Decreasing mitochondrial fission prevents cholestatic liver injury. J Biol Chem 2014, 289:34074-34088

34. Barber VC, Wright DE: The fine structure of the eye and optic tentacle of the mollusc Cardium edule. J Ultrastruct Res 1969, 26:515-528

35. Chertov AO, Holzhausen L, Kuok IT, Couron D, Parker E, Linton JD, Sadilek M, Sweet IR, Hurley JB: Roles of glucose in photoreceptor survival. J Biol Chem 2011, 286:34700-34711

36. Sies H: Role of metabolic $\mathrm{H} 2 \mathrm{O} 2$ generation: redox signaling and oxidative stress. J Biol Chem 2014, 289:8735-8741

37. Cherubini M, Ginés S: Mitochondrial fragmentation in neuronal degeneration: toward an understanding of HD striatal susceptibility. Biochem Biophys Res Commun 2017, 483:1063-1068 